\title{
The Role of Mass Eccentricity on the Earthquake Induced Torsion in Buildings
}

\author{
George K. Georgoussis ${ }^{1}$, Anna Mamou ${ }^{1}$ \\ ${ }^{I}$ Department of Civil Engineering Educators, School of Pedagogical and Technological Education (ASPETE), N. Heraklion 14121, \\ Attica, Greece. \\ *Corresponding author E-mail: ggeorgo@tee.gr
}

\begin{abstract}
This paper investigates the effect of mass eccentricity on the earthquake induced torsion in buildings. An analytical solution is proposed, which identifies the location of a key structural element for which the torsional response of a structure is minimized for any height wise variation of the mass eccentricities. The accuracy of the analytical solution is then verified with parametric numerical modelling on 9story buildings with height wise variations of the accidental eccentricities. The numerical modeling results show that the top rotations and base torques have an inverted peak, which indicates an optimum location of the key structural element, for which the torsional response of the structure is minimized. The location of the key element which minimizes the torsional response of the structure predicted by the analytical solution is verified with reasonable accuracy by the numerical modeling results.
\end{abstract}

Keywords: Earthquake engineering, Mass eccentricity, Modal analysis, Optimum torsion axis

\section{Introduction}

Experimental tests, numerical modeling studies and observations from previous earthquakes have revealed that translational - torsional coupling can have a deleterious effect on the structural stability of buildings ([1],[2],[3],[4],[5],[6]). Current code provisions recommend that the effects of earthquake induced torsion should be introduced by the following pair of design eccentricities

$$
\begin{aligned}
& e_{d 1}=\alpha e_{s}+\beta b \\
& e_{d 2}=\gamma e_{s}-\beta b
\end{aligned}
$$

where $e_{s}$ is the static eccentricity, which is defined as the distance between the centre of rigidity $(\mathrm{CR})$ and the centre of mass $(\mathrm{CM})$ and the term $\pm \beta b$ represents the accidental eccentricity, where $b$ is the building plan dimension perpendicular to the direction of ground motion. The coefficients $\alpha$ and $\gamma$ are dynamic amplification factors, which are specified by individual country codes. In general, these factors vary from code to code (for example [7] requires that $\alpha=\gamma=1.0$, but in [8], $\alpha=1.0$ and $\gamma$ may take values less than unity when the static eccentricity is greater than the accidental, whereas the Greek seismic code [9] requires that $\alpha=1.5$ and $\gamma=0.5$ ), while the term of the accidental eccentricity is usually taken as a percentage (5\%-10\%) of the building plan dimension perpendicular to the direction of the ground motion $b$.

From Equation 1(a,b) it follows, that a reduced or even negligible static eccentricity, results in lower values of design eccentricities and minor torsional effects.. When $e_{s}=0$, the design eccentricities of Eq. $(1 \mathrm{a}, \mathrm{b})$ are reduced to static eccentricities, since the dynamic factors $\alpha$ and $\gamma$ only act as amplifiers of the static eccentricity. In cases, where the code provisions are based on Eq. $(1 \mathrm{a}, \mathrm{b})$, as in for example [7], the dynamic effects are not taken into account. For this reason it may be more reasonable to apply the dynamic amplification factor, $\alpha$, to the accidental eccentricity only, as for example in [8] where this factor, denoted as $A_{x}$, takes values between 1 and 3. It should be noted that in all the aforementioned codes, $\beta=0.05$. Nevertheless, the reason for defining the design eccentricities relative to the centre of rigidity (CR), is that in single-story systems with a rigid floor diaphragm, any lateral load passing through the CR causes only a translation of the slab and any torque applied on the slab causes only a rotation about the CR. The CR is therefore usually taken as the reference point to quantify the torsional effects on structural buildings. Whilst defining the $\mathrm{CR}$ in one-story systems may be a relatively straight forward procedure, as the CR is essentially the center of lateral stiffness, its definition, in the various floors of a multistory building may not be an equally straightforward task. In [10] the centers of rigidity (CRs) were defined as a set of points located at the floor levels, such that any distribution of lateral loading passing through the CRs, would only cause a translational movement of the floors. It is now widely accepted that using the centers of rigidity (CRs) as a reference system to assess the torsional response of buildings, poses a number of difficulties associated with the fact that the centers of rigidity are generally not located on the same vertical line and are also load dependent ([11], [12], [13], [14]).

An alternative reference system for implementing the torsional code provisions can be determined by locating an optimum tosional axis (OTA), for which any in-plane applied lateral load, the torsional distortion on the structure is minimized. The optimum torsional axis (OTA) may be defined as the axis passing through the stiffness centre of an equivalent single story system [15]. The stiffness centre of the equivalent single story system defines the modal centre of rigidity (m-CR) and its derivation is based on the approximate method of the continuous medium, which assumes uniform over the height building systems. It was demonstrated that for building structures, where the centers of mass of the vari- 
ous floors are located on the same vertical axis, their dynamic response is essentially translational, when the mass axis passes through the modal centre of rigidity (m-CR) ([16],[17],[18]). The modal centre of rigidity (m-CR) may be either defined using the approximate method of the continuous medium or using the discrete element approach (stiffness method) [19]. In a recent paper it was shown, that when the mass axis coincides with the OTA, this results in an essentially translational response, and such a structural configuration may be easily attained by a suitable arrangement of the lateral load resisting bents [19]. This paper examines how such an arrangement can also be achieved, when the centers of floor masses are shifted, in a random spatial way, from their nominal positions, but within the limits of the code [20]. The role of mass eccentricities on the torsional response of a building is investigated though an analytical (mathematical) approach and the analytical solution is then verified with a case study on 9-story buildings where the location of the accidental eccentricities is varied throughout the building height.

\section{Analytical Solution}

Assuming a N-story building with orthogonal framing along the global $\mathrm{x}$ and $\mathrm{y}$ directions and rigid floor slabs, which is subjected to the lateral load vector

$$
\mathbf{F}=\left\langle\begin{array}{lll}
\mathbf{f}_{x} & \mathbf{f}_{y} & \mathbf{f}_{\theta}
\end{array}\right\rangle^{\mathrm{T}}
$$

where the load sub-vectors $\mathbf{f}_{\mathrm{x}}, \mathbf{f}_{\mathrm{y}}$ and $\mathbf{f}_{\theta}$ are of $N$ order. The equilibrium equation between forces and displacements may be expressed by the following matrix equation

$$
\left[\begin{array}{ccc}
\mathbf{K}_{x x} & 0 & \mathbf{K}_{x z} \\
0 & \mathbf{K}_{y y} & \mathbf{K}_{y z} \\
\mathbf{K}_{z x} & \mathbf{K}_{z y} & \mathbf{K}_{z z}
\end{array}\right]\left\{\begin{array}{l}
\mathbf{u}_{x} \\
\mathbf{u}_{y} \\
\mathbf{u}_{\theta}
\end{array}\right\}=\left\{\begin{array}{l}
\mathbf{f}_{x} \\
\mathbf{f}_{y} \\
\mathbf{f}_{\theta}
\end{array}\right\}
$$

which displays a set of $3 N$ equations for the displacement vectors $\mathrm{u}_{\mathrm{x}}$ and $\mathrm{u}_{\mathrm{y}}$, and the rotation vector $\mathrm{u}_{\theta}$ (all of $N$ order) in an arbitrary coordination system Oxyz. For buildings which have lateral load resisting elements in two orthogonal directions, the sub-matrices of the above stiffness matrix may be expressed as

$$
\begin{aligned}
& \mathbf{K}_{x x}=\Sigma \mathbf{K}_{i} \\
& \mathbf{K}_{y y}=\Sigma \mathbf{K}_{j} \\
& \mathbf{K}_{x z}=\mathbf{K}_{z x} \mathrm{~T}=-\Sigma y_{i} \mathbf{K}_{i} \\
& \mathbf{K}_{y z}=\mathbf{K}_{z y}^{\mathrm{T}}=\Sigma x_{j} \mathbf{K}_{j} \\
& \mathbf{K}_{z z}=\Sigma x_{j}^{2} \mathbf{K}_{j}+\Sigma y_{i}^{2} \mathbf{K}_{i}
\end{aligned}
$$

where the element sub-matrices $\mathrm{K}_{i}$ and $\mathrm{K}_{j}($ of $N \mathrm{x} N$ order) are the stiffness matrices of the $\mathrm{i}$-bent (oriented along the $\mathrm{x}$-direction at a distance $y_{i}$ from the $\mathrm{x}$-reference axis) and the $\mathrm{j}$-bent (is oriented along the y-direction at a distance $x_{j}$ from y-reference axis) respectively.

Assuming that the structural building is subjected to a translational ground excitation along the y-direction, the requirement of a practically translational response implies that the applied lateral loads should be proportional to the first modal vector of the uncoupled structure, which is the dominant mode of vibration of a medium height building. That is:

$$
\mathbf{f}_{y}=\mathbf{M} \boldsymbol{\Phi}_{y 1}=\frac{1}{\omega_{\mathrm{y} 1}^{2}} \mathbf{K}_{y y} \boldsymbol{\Phi}_{y 1}
$$

where
$\mathbf{M}=\left[\begin{array}{llll}m_{1} & & & \\ & m_{2} & & \\ & & \cdot & \\ & & & m_{N}\end{array}\right]$

$\mathrm{M}$ is the diagonal mass matrix and $m_{1}, m_{2} \ldots m_{N}$ are the floor masses, numbered from the base upwards, and $\boldsymbol{\Phi}_{y 1}$ and $\boldsymbol{\omega}_{\mathrm{y} 1}$ are the first modal shape and frequency of the uncoupled structure. In such a case, the floor components of the lateral load vector $\mathbf{f}_{\mathrm{y}}$ are applied at the centres of mass of the various floors, which are located at a distance $e_{m x l}, e_{m x 2}, \ldots e_{m x N}$ from the vertical reference axis respectively. Therefore, since $\mathbf{f}_{\mathrm{x}}=\mathbf{0}$, the torsional moment vector about this axis will be equal to

$$
\mathbf{f}_{\theta}=\mathbf{E}_{m} \mathbf{f}_{y}
$$

Where $\mathrm{E}_{\mathrm{m}}$ is the eccentricity mass matrix defined as

$\mathbf{E}_{m}=\left[\begin{array}{llll}e_{m \times 1} & & & \\ & e_{m x 2} & & \\ & & \cdot & \\ & & & e_{m \times N}\end{array}\right]$

The displacement vectors $\mathrm{u}_{x}, \mathrm{u}_{y}$ and $\mathrm{u}_{\theta}$, derived from Equations (2b), may be expressed as

$\mathbf{u}_{x}=-\mathbf{K}_{x x}^{-1} \mathbf{K}_{x z} \mathbf{u}_{\theta}$

$\mathbf{u}_{y}=\mathbf{K}_{y y}^{-1} \mathbf{f}_{y}-\mathbf{K}_{y y}^{-1} \mathbf{K}_{y z} \mathbf{u}_{\theta}$

$\mathbf{u}_{\theta}=\mathbf{K}_{\theta \theta}^{-1}\left(\mathbf{E}_{m} \mathbf{M} \boldsymbol{\Phi}_{y 1}-\mathbf{K}_{z y} \mathbf{K}_{y y}^{-1} \mathbf{M} \boldsymbol{\Phi}_{y 1}\right)$

$\mathbf{K}_{\theta \theta}=\mathbf{K}_{z z}-\mathbf{K}_{z x} \mathbf{K}_{x x}^{-1} \mathbf{K}_{x z}-\mathbf{K}_{z y} \mathbf{K}_{y y}^{-1} \mathbf{K}_{y z}$

The displacement vectors $\mathrm{u}_{\mathrm{x}}, \mathrm{u}_{\mathrm{y}}$ and $\mathrm{u}_{\theta}$ are obtained by super positioning two loading conditions. The first loading condition is the response of the uncoupled structure (i.e. the system in which the floors are restrained against rotations) when it is subjected to a lateral force vector equal to $f_{y}$. This loading case provides the first term of the second part of Equation ( $7 b$ ). The second loading case constitutes a purely torsional moment vector, equal to

$$
\mathbf{T}_{\theta}=\mathbf{E}_{m} \mathbf{M} \boldsymbol{\Phi}_{y 1}-\mathbf{K}_{z y} \mathbf{K}_{y y}^{-1} \mathbf{M} \boldsymbol{\Phi}_{y 1}
$$

and its effect on the response of the uncoupled structure may be accounted for as a superposition of the effects of its modal components, $\mathrm{T}_{\theta n}(n=1,2, \ldots)$. This modal expansion of $\mathrm{T}_{\theta}$ is obtained as follows (Chopra, 2008):

$$
\mathbf{T}_{\theta}=\Sigma \mathbf{T}_{\theta n}=\Sigma \Gamma_{n} \mathbf{M} \Phi_{y n}(n=1,2, \ldots)
$$

where $\Phi_{y n}$ is the n-modal shape of the uncoupled structure and the corresponding modal participation factor $\Gamma_{n}$ is equal to

$$
\Gamma_{n}=\frac{\boldsymbol{\Phi}_{y n}^{T} \mathbf{T}_{\theta}}{\boldsymbol{\Phi}_{y n}^{\mathrm{T}} \mathbf{M} \boldsymbol{\Phi}_{y n}}
$$

If the first modal participation factor is set equal to zero $\left(\Gamma_{l}=0\right)$, this implies that the first modal component of $\mathrm{T}_{\theta}$, which represents its major contribution on the response of the assumed structure, is nullified and therefore the overall torsional effect is minimized. The $\Gamma_{1}=0$ condition, in combination with Equations (4a) and (9), implies that 


$$
\boldsymbol{\Phi}_{y 1}^{T} \mathbf{E}_{m} \mathbf{M} \boldsymbol{\Phi}_{y 1}=\boldsymbol{\Phi}_{y 1}^{T} \mathbf{K}_{z y} \mathbf{K}_{y y}^{-1} \mathbf{M} \boldsymbol{\Phi}_{y 1}=\frac{1}{\omega_{y 1}^{2}} \Sigma x_{j} \boldsymbol{\Phi}_{y 1}^{T} \mathbf{K}_{j} \boldsymbol{\Phi}_{y 1}
$$

Any structural configuration (any arrangement of the lateral load resisting bents) that satisfies the above equation is expected to undergo minimum torsional response. Dividing all parts of Equation (12a) by the first mode generalized mass, the following expression is obtained

$$
\frac{\boldsymbol{\Phi}_{y 1}^{T} \mathbf{E}_{m} \mathbf{M} \boldsymbol{\Phi}_{y 1}}{\boldsymbol{\Phi}_{y 1}^{T} \mathbf{M} \boldsymbol{\Phi}_{y 1}}=\frac{1}{\omega_{y 1}^{2}} \sum x_{j} \frac{\boldsymbol{\Phi}_{y 1}^{T} \mathbf{K}_{j} \boldsymbol{\Phi}_{y 1}}{\boldsymbol{\Phi}_{y 1}^{T} \mathbf{M} \boldsymbol{\Phi}_{y 1}}
$$

An approximate expression of Equation (12b) may be given by using the concept of the element frequency of the lateral load resisting elements [15]. For example, for the j-element (bent), its element frequency, $\omega_{j 1}$, is defined as

$$
\omega_{j 1}^{2}=\frac{\boldsymbol{\Phi}_{j 1}^{\mathrm{T}} \mathbf{K}_{j} \boldsymbol{\Phi}_{j 1}}{\boldsymbol{\Phi}_{j 1}^{\mathrm{T}} \mathbf{M} \boldsymbol{\Phi}_{j 1}}
$$

and represents the first mode frequency of the j-lateral load resisting element, when it is assumed to carry, as a plane frame, the mass per floor of the real structure $\left(\Phi_{j 1}\right.$ is the first mode vector of the particular j-bent). It is worth noting that a lower bound of the first modal frequency of the uncoupled structure, $\omega_{y 1}$, may be evaluated by means of the element frequencies, according to Southwell's formula [21]. For example, if the lateral stiffness in the $\mathrm{y}$-direction is composed by a number of k-elements, then

$$
\omega_{y 1}^{2} \approx \sum_{j=1}^{k} \omega_{j 1}^{2}
$$

The equation above is based on Rayleigh's quotients, according to which any approximate first mode shape vector may provide a reasonable estimate of the first mode frequency. Replacing each mode vector $\Phi_{y 1}$ with $\Phi_{j 1}$, in the second part of Equation (12b) equation (12b) may be rewritten as

$$
\frac{\boldsymbol{\Phi}_{y 1}^{T} \mathbf{E}_{m} \mathbf{M} \boldsymbol{\Phi}_{y 1}}{\boldsymbol{\Phi}_{y 1}^{T} \mathbf{M} \boldsymbol{\Phi}_{y 1}}=\frac{1}{\omega_{y 1}^{2}} \Sigma x_{j} \omega_{j 1}^{2}
$$

where the fundamental frequency of the uncoupled system, $\omega_{\mathrm{y} 1}$, may alternatively also be obtained from Equation (14).

When all the floor masses are aligned on a vertical (mass) axis, i.e.:

$$
e_{m x l=} e_{m x 2=\cdots=} e_{m x N=} e_{m x}
$$

which suggests that the eccentricity matrix of Equation (6) is equal to

$$
\mathbf{E}_{m}=e_{m x} \mathbf{I}
$$

where I is the unit matrix, Equation (15) may be expressed as:

$e_{m x}=\frac{\sum x_{j} \omega_{j 1}^{2}}{\omega_{y 1}^{2}}$

The last part of equation (17a) defines the optimum torsional axis (OTA) [19]. When the OTA coincides with the mass axis, the torsional response is minimized. By definition, the coordinates of the OTA depend on the structural member frequencies and different structural configurations may result in OTA axes that coincide with the mass axis. When the mass axis is taken as the reference axis, the condition for minimum torsional response requires that the arrangement of the various bents should satisfy the following equation

$$
\sum x_{j} \omega_{j 1}^{2}=0
$$

In structural engineering practice and due to architectural or functional considerations, the structural design engineer may need to relocate the lateral load resisting bents, to obtain a structural configuration, for which the OTA coincides with the mass axis. In practice, it is convenient to construct such a system by locating a particular element (denoted as key element and assumed to be the $\mathrm{k}$-element, when the lateral resistance is provided by a number of $\mathrm{k}$ bents), in a way that

$$
\sum_{j=1}^{k} x_{j} \omega_{j 1}^{2}=\sum_{j=1}^{k-1} x_{j} \omega_{j 1}^{2}+x_{k} \omega_{k 1}^{2}=0
$$

In buildings where the centres of floor masses are not aligned on a vertical line, the vertical axis of the reference system may be taken as the axis passing through the centre of the total mass of the building structure. That is, with respect to the initially assumed Oxyz coordination system, the x-coordinate, $e_{m x}$, and the vertical reference axis may be defined as

$e_{m x} M_{t o t}=\Sigma e_{m x n} m_{n} \quad n=1,2, \ldots N$

where $M_{\mathrm{tot}}$ is the total mass of the building $\left(=m_{1}+m_{2}+\ldots+m_{N}\right)$. The new vertical reference axis, defined by Equation (19), has the advantage that when the gravity loads are uniformly distributed on all the floors and when their centroids are located on the same vertical line, the aforementioned axis is the mass axis of the system. In such a case, when the code accidental eccentricities are neglected, any structural configuration that satisfies Equation (17b) defines a system of minimum torsional response. When however, the accidental eccentricities are taken into account, the optimum location of the k-element may be determined by evaluating the first term of Equation (15). A convenient method to evaluate this part of Equation (15) is to express the matrix product $\mathrm{E}_{m} \mathrm{M}$ as :

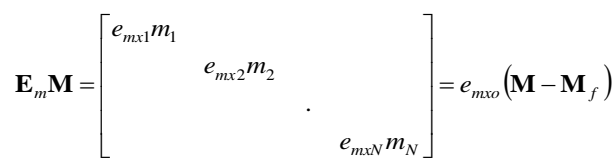

where $\mathbf{M}_{f}$ is a matrix defined as

$\mathbf{M}_{f}=\left[\begin{array}{cccc}\left(1-e_{m \times 1} / e_{m \times o}\right) m_{1} & & & \\ & \left(1-e_{m \times 2} / e_{m \times o}\right) m_{2} & & \\ & & \cdot & \\ & & \left(1-e_{m \times N} / e_{m \times o}\right) m_{N}\end{array}\right]=\left[\begin{array}{llll}m_{f 1} & & & \\ & m_{f 2} & \\ & & \cdot & \\ & & & m_{f N}\end{array}\right]$

and $e_{m x o}$ is the largest, in absolute value, of all floor mass eccentricities, i.e.:

$\left|e_{m x o}\right| \geq\left|e_{m x n}\right|, \quad \mathrm{n}=1,2 \ldots N$

It is worthwhile noting that none of the $\mathrm{M}_{f}$ elements registers a negative value and therefore this matrix may be considered as a fictitious mass matrix. The sum of the fictitious floor masses of $\mathrm{M}_{f}$ is equal to the total mass $M_{\mathrm{tot}}$ of the assumed building, since the location of the vertical reference axis satisfies Equation (19). Rearranging Equation (15), in combination with Equations (20a) and (20b), the following is obtained:

$e_{x m o}(1-R G M)=\frac{1}{\omega_{y 1}^{2}}\left(\sum_{j=1}^{k-1} x_{j} \omega_{j 1}^{2}+x_{k} \omega_{k 1}^{2}\right)$ 
where, RGM is defined as the ratio of the generalized masses and is equal to:

$$
R G M=\frac{\boldsymbol{\Phi}_{y 1}^{T} \mathbf{M}_{f} \boldsymbol{\Phi}_{y 1}}{\boldsymbol{\Phi}_{y 1}^{T} \mathbf{M} \boldsymbol{\Phi}_{y 1}}
$$

Which may be also alternatively expressed as

$$
R G M=\frac{\boldsymbol{\Phi}_{y 1}^{T} \mathbf{K}_{y y} \boldsymbol{\Phi}_{y 1} / \boldsymbol{\Phi}_{y 1}^{T} \mathbf{M} \boldsymbol{\Phi}_{y 1}}{\boldsymbol{\Phi}_{y 1}^{T} \mathbf{K}_{y y} \boldsymbol{\Phi}_{y 1} / \boldsymbol{\Phi}_{y 1}^{T} \mathbf{M}_{f} \boldsymbol{\Phi}_{y 1}}
$$

In this equation, the numerator defines the square value of the first mode frequency, $\omega_{y l}$, of the uncoupled structure, while the denominator, defined as

$$
R Q=\frac{\boldsymbol{\Phi}_{y 1}^{T} \mathbf{K}_{y y} \boldsymbol{\Phi}_{y 1}}{\boldsymbol{\Phi}_{y 1}^{T} \mathbf{M}_{f} \boldsymbol{\Phi}_{y 1}}
$$

is a Rayleigh's quotient of the fundamental frequency, $\omega_{y f 1}$, of the uncoupled system which has the floor masses shown in the main diagonal of the matrix of Equation (20b). Therefore, since it is easy to evaluate $\omega_{y f 1}$ by any structural analysis software, an approximate equation which provides the required location of the $\mathrm{k}$ element, $x_{k}$, is as follows

$$
e_{x m o}\left(1-\frac{\omega_{y 1}^{2}}{\omega_{y f 1}^{2}}\right)=\frac{1}{\omega_{y 1}^{2}}\left(\sum_{j=1}^{k-1} x_{j} \omega_{j 1}^{2}+x_{k} \omega_{k 1}^{2}\right)
$$

It should be noted that when the maximum mass eccentricity appears on two floors with opposite algebraic sign, the eccentricity term $e_{m x o}$ in equations (20a) and (21a) should be taken as that of the lower floor. To explain this suggestion [20] let assume here that the accidental floor eccentricities of a given structure are equal in absolute values, but those in the upper half (assumed for example positive) are of opposite algebraic sign of the lower part (assumed negative). It may be worth recalling that the sum of the fictitious floor masses of Equation (20b) is equal to the total mass $M_{\mathrm{tot}}$. Therefore, when $e_{m x o}$ is taken as that of the floors in the upper half of its height, this means that the fictitious uncoupled system, which specifies $\omega_{y f 1}$, has masses (of double value) only in the lower half of its height. The value of $\omega_{y f 1}$, thus produced, is equal to

$$
\omega_{f y 1}^{2}=\frac{\boldsymbol{\Phi}_{f y 1}^{T} \mathbf{K}_{y y} \boldsymbol{\Phi}_{f y 1}}{\boldsymbol{\Phi}_{f y 1}^{T} \mathbf{M}_{f} \boldsymbol{\Phi}_{f y 1}}
$$

where $\Phi_{f y l}$ is the first mode vector of the aforementioned fictitious uncoupled system. There is a clear difference between the shape functions $\Phi_{y l}$ (in Equation (22)) and $\Phi_{f y l}$ (in Equation (24). Both of them satisfy the displacement boundary conditions at the base of the structure, but $\Phi_{y l}$ satisfies the force boundary conditions at the top of the system, while $\Phi_{f y l}$ satisfies the corresponding conditions at the mid height of the system, since there are no masses above this point. Therefore, the procedure of approximating the quotient $R Q$ of Equation (22), with the frequency of Equation (24) may not be reasonable. When $e_{m x o}$ is taken as that of the lower floors, the corresponding fictitious uncoupled system, which has (double) masses only in its top part, provides the first mode frequency $\omega_{y f 1}$ by an expression similar to Equation (24), but the corresponding mode vector $\Phi_{f y l}$ satisfies now the force boundary conditions at the top of the system, as the vector $\Phi_{y l}$. Therefore, it is reasonable to approximate the quotient $R Q$ of Equation (22), by the square value of the first modal frequency of the uncoupled fictitious system

\section{Case study}

The plan view of the three analyzed concrete building models T0/B9, T3/B6 and T6/B3 is presented in Fig.1. All three models comprise 9-storey mono-symmetrical building systems, with in plan and in elevation irregularities. T0/B9 has nine identical orthogonal floors of $23 \times 16 \mathrm{~m}$, as shown by the exterior perimeter. T3/B6 is a setback building, which comprises a six floor base structure, with plan dimensions of $23 \times 16 \mathrm{~m}$ (shown by the exterior perimeter), and a three floor top structure with a reduced size of $18 \times 12 \mathrm{~m}$ (indicated by the interior perimeter in Fig. 1). Model T6/B3 is also a setback building, comprising a base structure with three floors identical to those of Model T0/B9, and a top structure comprising six floors similar to the top floors of Model T3/B6.

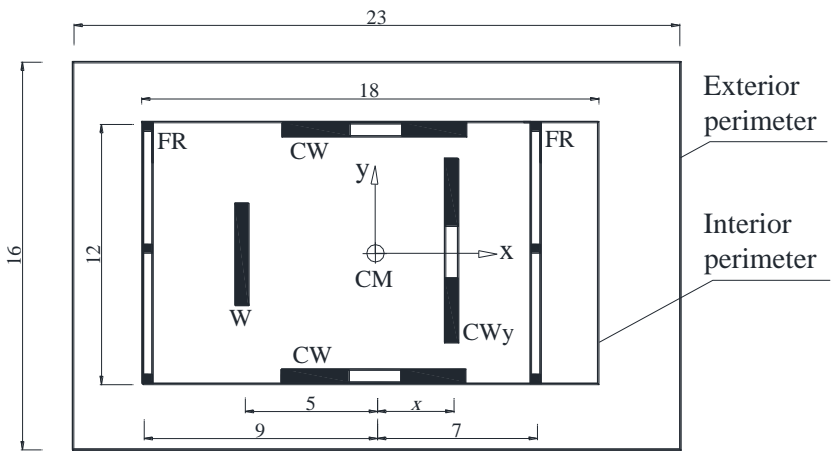

Fig. 1: Plan view of the analysed building models (all dimensions in meters)

In all three building models, the lateral load resisting system along the $\mathrm{y}$-direction consists of a wall, $\mathrm{W}$, a coupled wall bent, $\mathrm{CWy}$, and by two moment resisting frames, FR. Two coupled wall bents provide lateral resistance in the $\mathrm{x}$-direction. When uniformly distributed gravity loads are assumed on the floor slabs, their centres of mass lie on the same vertical axis (nominal location of the Mass Axis). Full details of these concrete models may be seen in [20].

For each building model T0/B9, T3/B6 and T6/B3, six different mass eccentricity configurations were investigated. The configuration of the first three analyzed mass eccentric systems of building models T0/B9, T3/B6 and T6/B3 are shown in Fig. 2. In all systems the centers of mass $(\mathrm{CM})$ of the base and top structure were shifted along the $\mathrm{x}$-axis as shown in the aforementioned Figure, but in all cases the center of the total mass, $M_{t o t}$, lies on the vertical (nominal) axis passing through the centroids of all floors. Since all the three analyzed mass eccentricities systems for each building model (in the case of T3/B6 the eccentric systems (+)A:T3/B6, (+)B:T3/B6 and (+)C:T3/B6)) are structurally asymmetric along the $\mathrm{y}$-direction, all the aforementioned systems were also investigated for the reversed location of the various floor eccentricities (i.e.: their algebraic sign was reversed, (-)A:T3/B6, $(-) B: T 3 / B 6$ and (-)C:T3/B6). Similarly, the first three eccentric systems of model T6/B3, are (+)A:T6/B3, (+)B:T6/B3 and $(+) \mathrm{C}: \mathrm{T} 6 / \mathrm{B} 3$ and the reversed eccentricities are (-)A:T6/B3, (B:T6/B3 and (-)C:T6/B3.
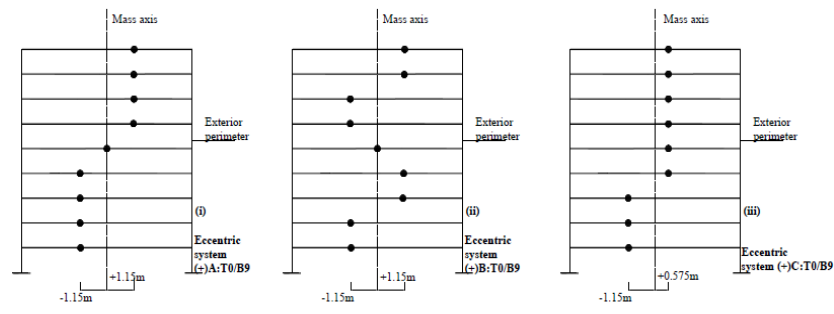

Fig. 2a: The first three analyzed mass eccentric systems of model T0/B9: (i) (+)A:T0/B9; (ii) (+)B:T0/B9 and (iii) (+)C:T0/B9 

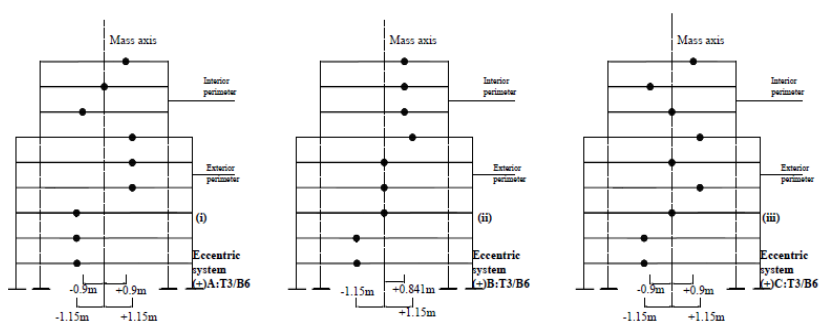

Fig. 2b: The first three analyzed mass eccentric systems of model T3/B6: (i) (+)A:T3/B6; (ii) (+)B:T3/B6 and (iii) (+)C:T3/B6
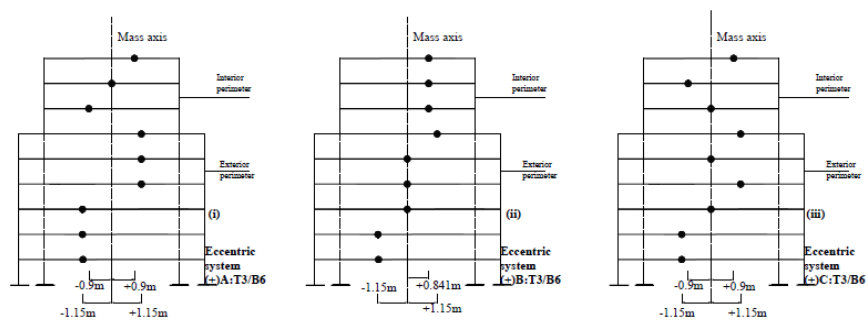

Fig. 2c The first three analyzed mass eccentric systems of model T6/B3: (i) (+)A:T6/B3; (ii) (+)B:T6/B3 and (iii) (+)C:T6/B3

\section{Results and Discussion}

The numerical analysis of the mass eccentricity configurations for each building model T0/B9, T3/B6 and T6/B3 was performed with the structural analysis program SAP2000-V16, for a ground excitation along the y-direction, as defined by the acceleration spectrum of EC8-2004 (type 1, ground type B, soil factor 1, horizontal ground acceleration $0.40 \mathrm{~g}$ ). The normalized base torques $\overline{\mathrm{T}}=\mathrm{T} / \mathrm{r}_{\mathrm{b}} \mathrm{V}_{\mathrm{o}}$ (where $\mathrm{V}_{\mathrm{o}}$ is the base shear of the corresponding uncoupled building model and $r_{b}$ is the radius of gyration of the floor mass of the base structure) and top rotations, $\Theta$, for the different locations (indicated by the normalized coordinate $\bar{x}=x / \mathrm{r}_{\mathrm{b}}$ ) of the coupled wall bent $\mathrm{CWy}$, of the systems of Figs. 2a-2c are shown by the red lines in Fig. 3. All the data were calculated on the basis of the first 12 peak modal values combined according to the CQC rule (the damping ratio in each mode of vibration was taken as $5 \%)$.

The blue lines in Fig. 3 show the torsional response of the systems with reversed mass eccentricities, while the black lines show the response of these systems when no mass eccentricities are taken into account. The results in Fig. 3 show that the variation of base torques is smoother than those of the top rotations, which suggests that the mostly affected response parameter is the top rotation. The inverted peaks of the top rotation and, to a lesser degree, of the base torques, clearly indicate an optimum location of the coupled wall bent CWy. In Figures $3 \mathrm{a}$ and $3 \mathrm{~b}$ the minimum values of the rotations and base torque point to almost the same value of $\bar{x}$. This is less noticeable by the torques curves (solid lines) in Figure 3c. They show a rather extended range of locations of the coupled wall bent $\mathrm{CWy}$ where the base torque registers small values.

The inverted peaks of the base torques and top rotations are pointing to almost symmetrical locations with respect to those indicated by the black lines, whose inverted peaks indicate the optimum location of CWy where the torsional response is minimized. The results demonstrate, that, for any spatial variation of mass eccentricities, the optimum location of the key element, which minimizes the torsional effect of the structure has an almost symmetrical location, with respect to its nominal location, when the mass eccentricities are reversed. Figure 4 shows the locations of the CWy bent, which minimizes the torsional response of the six mass eccentricity configurations, as predicted by the analytical solution [20]. The locations of the models without mass eccentricities, as derived in [19], are also shown Figure 4. The results suggest that the numerical modeling verifies the analytical solution with reasonable accuracy.

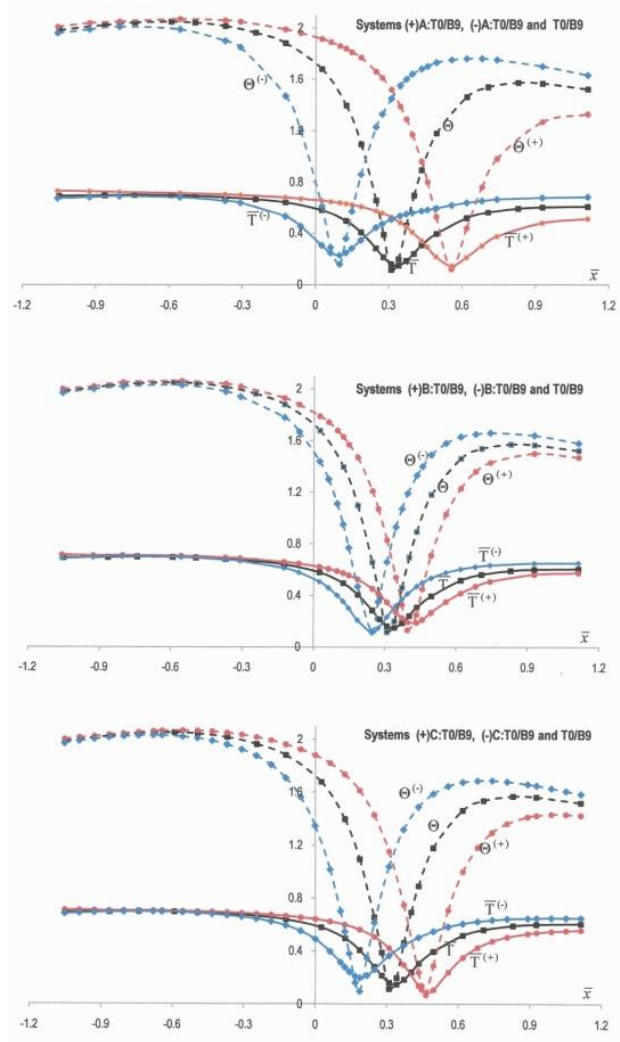

Fig. 3a: Top rotations $\left(\times 10^{-2}\right.$ rads) and normalized base torques of the eccentric systems $(+) \mathrm{A}: \mathrm{T} 0 / \mathrm{B} 9,(+) \mathrm{B}: \mathrm{T} 0 / \mathrm{B} 9$ and $(+) \mathrm{C}: \mathrm{T} 0 / \mathrm{B} 9$ red lines) and the systems (-)A:T0/B9, (-)B:T0/B9 and (-)C:T0/B9 (blue lines), together with the corresponding quantities of the system with no mass eccentricities (model T0/B9, black lines)

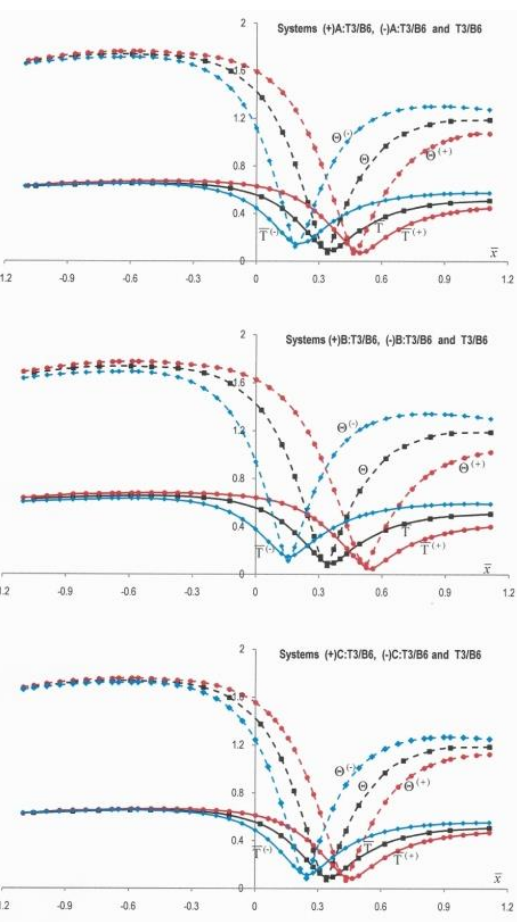

Fig. 3b: Top rotations $\left(\times 10^{-2}\right.$ rads) and normalized base torques of the eccentric systems (+)A:T3/B6, (+)B:T3/B6 and (+)C:T3/B6 (red lines) and the systems (-)A:T3/B6, (-)B:T3/B6 and (-)C:T3/B6 (blue lines), together with the corresponding quantities of the system with no mass eccentricities (model T3/B6, black lines) 


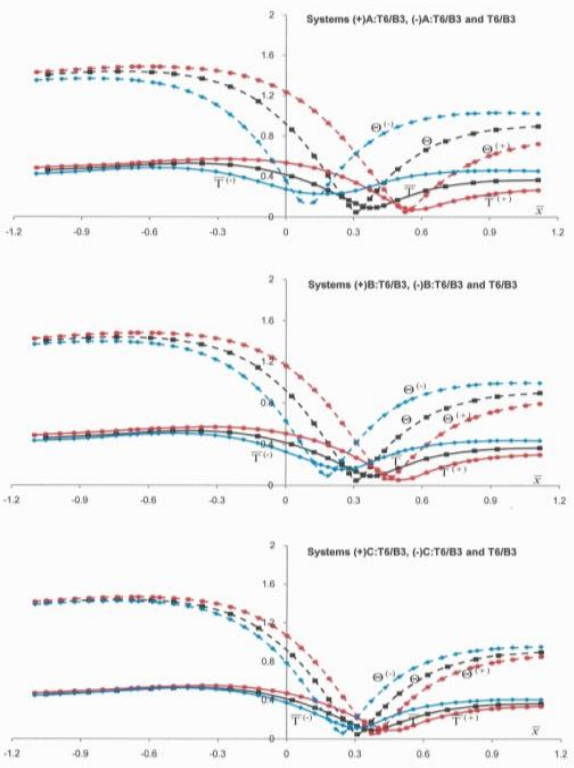

Fig. 3c: Top rotations $\left(\mathrm{x} 10^{-2}\right.$ rads) and normalized base torques of the eccentric systems (+)A:T6/B3, (+)B:T6/B3 and (+)C:T6/B3 (red lines) and the systems (-)A:T6/B3, (-)B:T6/B3 and (-)C:T6/B3 (blue lines), together with the corresponding quantities of the system with no mass eccentricities (model T6/B3, black lines)

\begin{tabular}{|c|c|c|c|}
\hline $\begin{array}{c}\text { Model with no } \\
\text { mass eccentricities }\end{array}$ & $\begin{array}{l}\text { Minimum torsional response for } \\
\text { CW } y \text { at } x^{\circ}\end{array}$ & $\begin{array}{c}\text { Mass eccentric } \\
\text { systems }\end{array}$ & $\begin{array}{l}\text { Minimum torsional response for } \\
\mathrm{CW} y \text { at } x^{+} / x^{-}\end{array}$ \\
\hline \multirow{5}{*}{ то/B9 } & \multirow{5}{*}{$x^{0}=0.248$} & (+)A:T0/B9 & $x^{*}=0.492$ \\
\hline & & (-)А:ТО/B9 & $x^{-}=0.004$ \\
\hline & & (+)B:T0/B9 & $x^{+}=0.341$ \\
\hline & & (-)В:Товя9 & $x^{-}=0.155$ \\
\hline & & $(+)$ C:T0/B9 & $x^{*}=0.391$ \\
\hline \multirow{6}{*}{ T3/B6 } & \multirow{6}{*}{$x^{0}=0.256$} & (-)С:То/B9 & $x^{-}=0.106$ \\
\hline & & (+)A:T3/B6 & $x^{*}=0.390$ \\
\hline & & $\frac{(-) \text { A:T3/B6 }}{(+) \text { B:T3/B6 }}$ & $x^{-}=0.120$ \\
\hline & & $\begin{array}{l}(+) \mathrm{B}: \mathrm{T} 3 / \mathrm{B} 6 \\
(-) \mathrm{B}: \mathrm{T} 3 / \mathrm{B} 6\end{array}$ & $\begin{array}{l}x^{+}=0.437 \\
x^{-}=0.074\end{array}$ \\
\hline & & $(+)$ C:T3/B6 & $\begin{array}{l}x=0.074 \\
x^{+}=0.350\end{array}$ \\
\hline & & $(-)$ C:T3/B6 & $x^{-}=0.160$ \\
\hline \multirow{6}{*}{$\mathrm{T} 6 / \mathrm{B}_{3}$} & \multirow{6}{*}{$x^{0}=0.246$} & (+)A:T6/B3 & $x^{+}=0.456$ \\
\hline & & $(-) \mathrm{A}: \mathrm{T} 6 / \mathrm{B} 3$ & $x^{-}=0.037$ \\
\hline & & $(+) \mathrm{B}: \mathrm{T} 6 / \mathrm{B} 3$ & $x^{+}=0.374$ \\
\hline & & $(-) \mathrm{B}: \mathrm{T} 6 / \mathrm{B} 3$ & $x^{-}=0.120$ \\
\hline & & (+)C:T6/B3 & $x^{*}=0.319$ \\
\hline & & $(-) \mathrm{C}: \mathrm{T} 6 / \mathrm{B} 3$ & $x^{-}=0.174$ \\
\hline
\end{tabular}

Fig. 4: Predicted normalized locations of the key element (CWy bent) for optimum torsional response of the systems of Figures $3 \mathrm{a}, \mathrm{b}, \mathrm{c}$ and the models with no mass eccentricity.

\section{Conclusions}

This paper investigates the effect of mass eccentricity on the earthquake induced torsion in buildings, using both analytical and numerical modelling results. An analytical solution is proposed, which identifies the optimum location of a key structural element for which the torsional response of a structure is minimized for any height wise variation of the mass eccentricities. The accuracy of the proposed analytical solution is then verified with numerical modeling on 9-story buildings with height wise variations of the accidental eccentricities.

The numerical modelling results show, that the variation of the base torques is smoother than those of the top rotations, which implies that the top rotations was the mostly affected response parameter. Both the top rotations and base torques have an inverted peak, which indicates an optimum location of the key structural element. Small shifts of the key element from its optimum location result in rather large torsional distortions, but these effects gradually become less significant as the key element moves further away from its optimum location. The results demonstrate that, for any spatial variation of mass eccentricities, the optimum location of the key structural element, has an almost symmetrical location, with respect to its nominal location, when the mass eccentricities are reversed. The location of the key element which minimizes the torsional response of the structure predicted by the analytical solution is verified with reasonable accuracy by the numerical modeling results.

\section{Acknowledgement}

The authors acknowledge the financial support for the dissemination of this work from the Special Account for Research of ASPETE through the funding program "Strengthening ASPETE'S RESEARCH", project "torsional response of building structures under seismic excitations".

\section{References}

[1] Kan, C.L., Chopra, A.K. (1977). Effects of torsional coupling on earthquake forces in buildings. J. Struct. Div. ASCE 103, No. 4, 805-819.

[2] Tso, W.K., Dempsey, K.M. (1980). Seismic torsional provision for dynamic eccentricity. Earthquake Eng Struct Dyn. 8, No. 3, 275 289.

[3] Dempsey, K.M., Irvine, H.M. (1979). Envelopes of maximum seismic response for a partially symmetric single-storey- building model. Earthquake Eng. Struct. Dyn. 7, No. 2, 161-180.

[4] Chandler, A.M., Hutchinson, G.L. (1986). Torsional coupling effects in the earthquake response of asymmetric building. Eng. Struct. 8, No. 4, 222-236.

[5] Jennings, P.C., Kuroiwa, J.H. (1968). Vibration and soil structure interaction tests of a nine-storey reinforced concrete building, Bull. Seism. Soc. Am. 58, No. 3, 891-916.

[6] Naeim F, Lew M. The (2000). 1999 earthquake disasters worldwide: how many times do we have to re- learn the fundamentals of seismic engineering? The Structural Design of Tall Buildings 9, No. 2, $161-182$.

[7] CEN. EN 1998-1 (2004). EuroCode 8: design of structures for earthquake resistance-part 1: general rules, seismic actions and rules for buildings. European Committee for Standardization, Bruxelles

[8] ASCE/SEI 7-10, Minimum Design Loads and Associated Criteria for Buildings and Other Structures, Committee on Minimum Design Loads for Buildings and Other Structures of the Codes and Standards Activities Division of the Structural Engineering Institute of ASCE

[9] EAK-2000. Greek Aseismic Code (2000) Greek Ministry of Environment, City Planning and Public Works. Greece.

[10] Cheung, VW.T., Tso, W.K. (1986). Eccentricity in irregular multistory building, Can. J. Civ. Eng 13, No. 1, 46-52.

[11] Makarios, T., Anastassiadis, K. (1998a). Real and fictitious elastic axis of multi-storey buildings applications. Struct. Design Tall Build. 7, No. 1, 33-45.

[12] Makarios, T., Anastassiadis, K., (1998b). Real and Fictitious Elastic axis of Multi-Storey Buildings Applications. Struct. Design Tall Build. 7, No. 1, 57-71.

[13] Marino, E.M., Rossi, P.P. (2004). Exact evaluation of the Location of the Optimum Torsion Axis. Struct. Design Tall Spec. Build. 13, No. 4, 277-290.

[14] Basu, D., Jain, S.K. (2007). Alternative Method to Locate Center of Rigidity in Asymmetric Buildings. Earthquake Engng. Struct. Dyn. 36, No. 7, 965-973.

[15] Georgoussis, G.K. (2016). An approach for minimum rotational response of medium-rise asymmetric structures under seismic excitations. Advances in Structural Engineering 19, No. 3, 420-436.

[16] Georgoussis, G.K. (2010). Modal Rigidity Center: its use for assessing elastic torsion in asymmetric buildings. Earthquakes and Structures 1, No. 2, 163-175.

[17] Georgoussis, G.K. (2014). Modified seismic analysis of multistory asymmetric elastic buildings and suggestions for minimizing the rotational response. Earthquakes and Structures 7, No. 1, 39-52.

[18] Georgoussis, G.K. (2015). Minimizing the torsional response of inelastic multistory buildings with simple eccentricity. Canadian J. Civil Engng 42, No. 11, 966-969.

[19] Georgoussis, G.K. (2017). Locating optimum torsion axis in asymmetric buildings subjected to seismic excitation. Proceedings of the Institution of Civil Engineers - Structures and Buildings, Ahead of Print, https://doi.org/10.1680/jstbu.17.00068

[20] Georgoussis, G.K., Mamou, A. (2018). The effect of mass eccentricity on the torsional response of building structures, under review by Structural Engineering and Mechanics, An International Journal

[21] Newmark, N.M., Rosenblueth, E. (1971). Fundamentals of Earthquake Engineering, Prentice Hall N.J. 